\title{
A new method of temporary plugging based on andreasen equation
}

\author{
Yuxue Sun, Yanyu Qin*, Qiming Li
}

Petroleum Engineering Department, Northeast Petroleum University, Daqing, China; *Corresponding Author: qinyanyu3737@126.com

Received 7 December 2010; revised 10 January 2011; accepted 13 January 2011.

\begin{abstract}
Based on the fractal nature of pore size in sandstone and grindingbody within a certain range and the Andreasen equation in theory, using the optimization theory of traditional temporary plugging agent for reference, we established a new temporary plugging method for reservoir protection. This new method emphasizes on the formulation and optimization of solid filling particles and softening particles. It is good in easy operation and strong applicability. Indoor experiments with the natural core from Hailaer area were conducted using this new method. Compared with traditional methods, the filtration reduced significantly and the permeability recovery was improved greatly, which verified the rationality of this new method.
\end{abstract}

Keywords: Temporary Plugging Technology; Formation Damage Prevention; Andreasen Equation

\section{INTRODUCTION}

In the early $1990 \mathrm{~s}$, based on " $1 / 3$ bridging rule" published by Abrams, Luo Pingya and Luo Xiangdong proposed "temporary plugging technology", which showed more effective through field application [1]. But the particle size selection of temporary plugging agent used in the technology was only base on whether the median diameter of temporary plugging agent matched the average pore throat diameter, which ignored many other factors in actual reservoir, such as complex pore throat structure, comprehensive size distribution and big pore throat that contributes more to the reservoir permeability. Many scholars both at home and abroad have studied this problem in theory $[2,3]$ Cui Yingchun et al. enriched the temporary plugging technology of fractal and Yan Jienian et al. enriched "the D90 rule" based on "Ideal Packing Theory". These two types of technology have achieved good results and have been proved its accuracy by field experiment.

However, the current temporary plugging methods also have some defects, such as, the distribution of temporary plugging particle size is narrow, and temporary plugging particles are mostly rigid. Thus there is greatly space to be increased. Based on previous theoretical basis, through optimizing, this paper choose 3 different kind of rigid temporary plugging particles and 1 kind of softening temporary plugging particle to mix together, then achieve a better filling effect $[4,5]$.

\section{THEORETICAL FOUNDATION}

As for formation protecting drilling fluid, "Ideal Packing Theory" means adding temporary plugging agent particles of continuous diameter size in accordance with the pore throat size to seal both the various pore throat in reservoir and the pore among plugging particles [6].

"Ideal Packing Theory" is base on fractal theory and holds that the pore in sandstone is self-similar in a certain extent, which has been confirmed by scholars home and abroad. At present, the main ingredient of conventional granular temporary plugging agents used in Chinese oil field is organic or anorganic material, most of which is made by grinding [7].

\subsection{Horsfield Model}

This model assumes that all particles is spherical, and spherical particles stacked at the same time have the same size. According to the order of accumulation, they are named as first balls, second balls, and by analogy, the following particles are stacked according to the six-party arrangement rules, of which the air voids is $25.94 \%$; second balls just enter surplus space of first time balls accumulation, other balls are also stacked. The corresponding computational results are showed in Table 1. As a result, if the diameter distribution is appropriate, the air voids can be reduced to an ideal level. 
Table 1. Results of Horsfield filling.

\begin{tabular}{cccc}
\hline Filling state & Ball radius & $\begin{array}{c}\text { Relative number } \\
\text { of balls }\end{array}$ & Air voids \\
\hline 1st balls & $\mathrm{r}_{1}$ & 1 & 0.2594 \\
2nd balls & $0.414 \mathrm{r}_{1}$ & 1 & 0.2070 \\
3rd balls & $0.225 \mathrm{r}_{1}$ & 2 & 0.190 \\
4th balls & $0.177 \mathrm{r}_{1}$ & 8 & 0.158 \\
5th balls & $0.116 \mathrm{r}_{1}$ & 8 & 0.149 \\
Filling materials & Tiny & Numerous & 0.039 \\
\hline
\end{tabular}

\subsection{Andreasen Equation}

Particles made by grinding are not uniform, but of varying sizes. Its distribution rule can not be described with Euclidean geometry. It has been proved to satisfy the fractal geometric property and keep the self-similar trait within a certain scope after studying. The mathematics foundation of this theory originates from Andreasen equation [8].

$$
U\left(D_{p}\right)=\left(D_{p} / D_{p l}\right)^{q}
$$

where $U\left(D_{p}\right)$ is cumulative sieve percentage, $D_{p}$ is current particle size, $D_{p l}$ is maximum particle size, $q$ is fuller index.

Andreasen thinks that various kinds of distributing air voids decreases with the minishing of distribution module $q$; when $q$ is within $0.33 \sim 0.50$, air voids is minimum; when $q$ is less than $1 / 3$, it is meaningless. This equation is consistent with the well-known G-S equation.

\section{A NEW METHOD OF TEMPORARY PLUGGING TECHNOLOGY}

\subsection{Proposal of the Method}

At present, the particle size distribution characteristics of conventional granular temporary plugging agent used in field is relatively standard. The range of the particle size is very narrow, which generally cannot meet the requirement of temporary plugging. The solution is to mix granular temporary plugging agent of three different particle size distributions according to a certain mixing ratio, which will make the temporary plugging effects dramatically increase, but when the granular temporary plugging agent is under the action of the positive differential pressure, the particles can only make bridge effect in the pore throat or in the fracture, so it is difficult to form effective seal by deformation. Meanwhile, the fragility of granular temporary plugging agent leads it liable to be ground finely during long cycle process, and then invade into the deep reservoir, so only when there is high solid content in the drilling fluid, can it make a temporary plugging effect. In addition, either triple filling or quadruple filling, and no matter how to adjust the particle size and gradation, it can not achieve zero filling of air void in accordance with Horsfield filling model. Therefore, beside formulating and optimizing three kinds of solid temporary plugging agents, this method also use soften deformational particles to completely seal reservoir.

\subsection{Step of Optimization}

- Choose representative sample for casting rock slice analysis or mercury injection experiments, and then measure maximum pore throat diameter in the reservoir.

- Draw the distribution graph of pore throat size changing with granular accumulative total volume percentage when fuller index $q$ equal $1 / 3$ and $1 / 2$, in which the enclosed area surrounded by the two curves is the area with minimum air void. If the cumulative distribution curve of the optimized temporary plugging particle is fell on the area with minimum air void, the sealing effect will become better along with the higher efficiency of particle accumulation.

- Based on 1/3 1/4 filling rules and other requirements (such as fluorescence, solubility, etc.), optimize softening deformational particles whose average grain diameter meet the requirements.

\section{INDOOR EXPRIMENT}

\subsection{Establishment of Model}

Verify the above theory and method through indoor experiment. The experimental cores are from the natural cores in Hailaer area, of which the maximum pore throat diameter of the reservoir is $26.5 \mu \mathrm{m}$ (i.e. $\mathrm{d} 90$ ), which is tested by mercury injection experiments. Make cumulative distribution curve template when the fuller index $\mathrm{q}$ equal $1 / 3$ and $1 / 2$, as is shown in Figure 1. This curve template is used for subsequent optimization of temporary plugging agent. Making blended temporary plugging curve between the two curves in template, then we get minimal porosity.

Take several kinds of normal rigid temporary plugging particles from field and use laser particle size analyzer to test particles size. The size distribution of rigid temporary plugging particles is showed in Table 2 . There are seven kinds of different temporary plugging agent in the table, including five kinds $\mathrm{CaCO}_{3}$ passing through sieves of different diameter; $\mathrm{WC}-1 \mathrm{C}$ is a kind of calcium, SAS is a softening shape-shifting particles; $d_{10}$ means ten percent particle size is less than the value, $d_{20}$ etc. is with the similar meaning. 


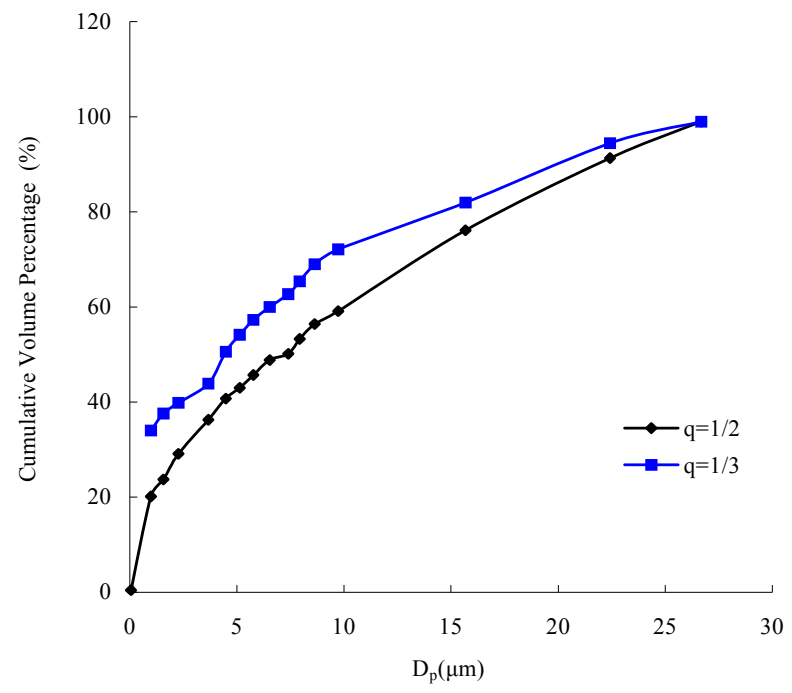

Figure 1. Template of cumulative distribution curve.

Table 2. Size distribution of rigid temporary plugging particles and flexible particles.

\begin{tabular}{cccccccc}
\hline \multirow{2}{*}{$\begin{array}{c}\text { Temporary } \\
\text { Plugging } \\
\text { Agent }\end{array}$} & \multicolumn{7}{c}{$\mathrm{CaCO}_{3}$ passing through sieves of } \\
\cline { 2 - 6 } & 0.050 & 0.030 & 0.025 & 0.015 & 0.005 & & \\
\hline$d_{10}$ & 0.36 & 0.48 & 0.96 & 1.14 & 0.47 & 11.4 & 1.25 \\
$d_{20}$ & 2.41 & 2.74 & 2.88 & 2.50 & 1.66 & 18.8 & 3.66 \\
$d_{30}$ & 8.22 & 7.39 & 8.64 & 4.93 & 2.17 & 32.66 & 6.52 \\
$d_{40}$ & 9.66 & 8.44 & 10.21 & 5.86 & 3.27 & 41.58 & 9.88 \\
$d_{50}$ & 14.67 & 11.25 & 12.32 & 7.24 & 4.40 & 48.34 & 12.34 \\
$d_{60}$ & 20.16 & 17.64 & 15.45 & 12.40 & 7.25 & 59.66 & 18.16 \\
$d_{70}$ & 28.69 & 22.33 & 20.66 & 16.21 & 9.33 & 77.82 & 22.31 \\
$d_{80}$ & 34.60 & 25.62 & 22.34 & 18.31 & 12.54 & 106.35 & 26.57 \\
$d_{90}$ & 41.57 & 30.28 & 25.66 & 19.67 & 14.86 & 141.60 & 32.50 \\
$d_{100}$ & 76.88 & 59.00 & 52.10 & 40.17 & 28.00 & 250.00 & 46.60 \\
\hline
\end{tabular}

By calculation and optimization experiment, We made the corresponding curve, and the filling curve is as Figure 2 shows. When temporary plugging proportion is $0.030 \mathrm{~mm}: 0.025 \mathrm{~mm}: 0.005 \mathrm{~mm}=25 \%: 45 \%: 30 \%$, the curve is between that of $q=1 / 2$ and $q=1 / 3$, then we obtain the best proportion and the air voids is minimal.

Considering the annular shearing wear of granular temporary plugging agent and the purpose of realizing zero air voids, we chose SAS, whose average particle size is $12.34 \mu \mathrm{m}$ and maximum diameter is $32.5 \mu \mathrm{m}$, as the preferred softening deformational particles in this experiment according to the optimization rule. By conducting formulation experiment with the above four temporary plugging particles, the completely filling curve is as Figure 3 shows. When the proportion of formulation is $0.030 \mathrm{~mm}: 0.025 \mathrm{~mm}: 0.005 \mathrm{~mm}$ : SAS $=20 \%: 45 \%$ :
$25 \%: 10 \%$, the curve is between that of $\mathrm{q}=1 / 2$ and $\mathrm{q}=$ $1 / 3$, then we obtain the best proportion and the air voids is minimal.

\subsection{Evaluating Test of Effect}

\subsubsection{Filtration Experiment}

By means of choosing organic silicon drilling fluid and adding proper amount of optimal shielding temporary plugging agent in it, we measured the API filtration and high temperature and high pressure filtration $\left(85^{\circ} \mathrm{C}\right.$ /3.5 MPa) to evaluate the sealing effect of this temporary plugging method.

Experimental data is showed in Table 3. The formula

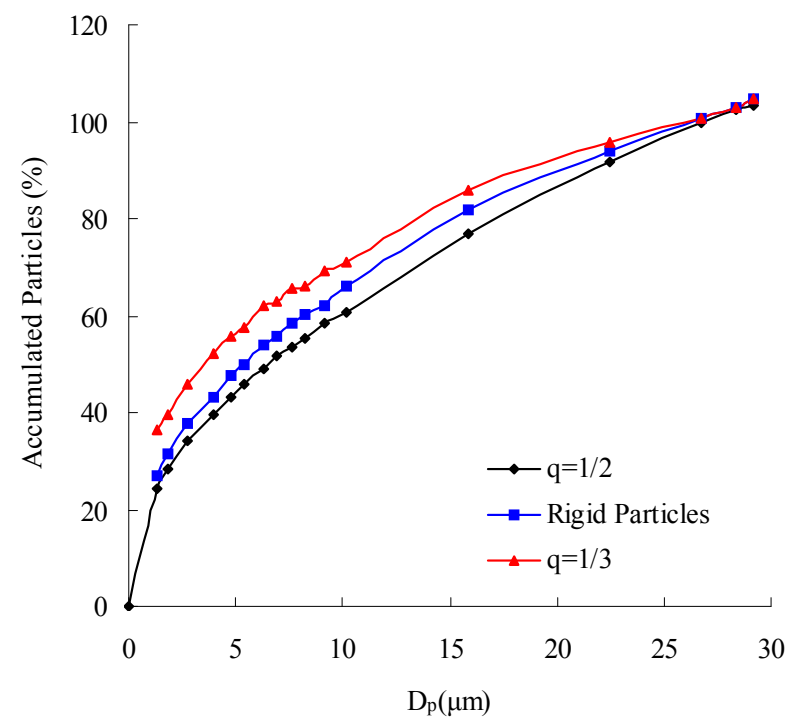

Figure 2. Filling state of rigid temporary plugging particles.

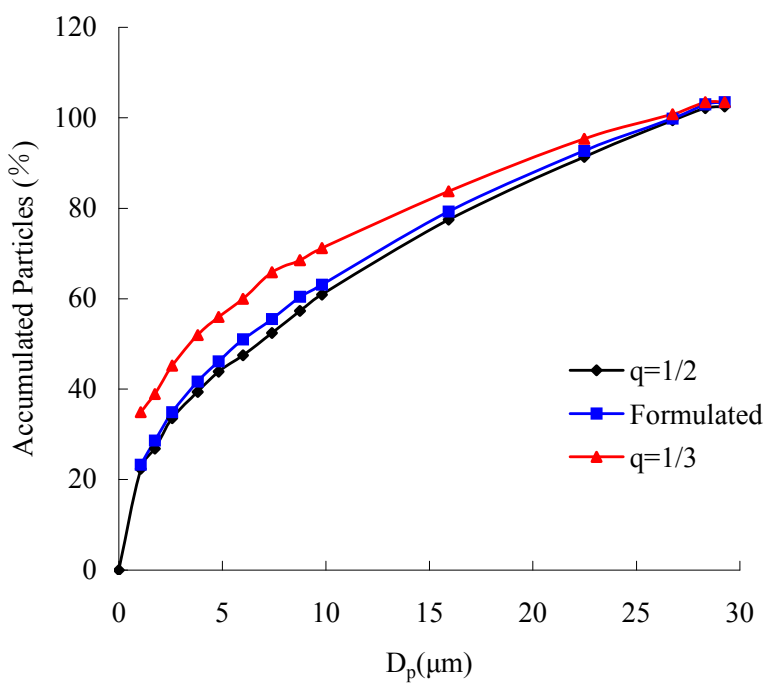

Figure 3. Filling state of formulated rigid temporary plugging particles. 
is as follow.

$6 \%$ bentonite $+0.5 \%$ soda $+0.2 \% \mathrm{KPA}+(0.7 \%-0.1 \%)$ ammonium salt $+(0.5 \%-1 \%)$ BTM- $2+1.5 \% \mathrm{CMJ}-2+1.5 \%$ PA-1

It can be seen from Table 3 that both the API filtration and HTHP filtration drops after adding temporary plugging agent, where which is added the temporary plugging agent based on Andreasen equation drops the most. This shows that the new temporary plugging method based on Ideal Packing Theory is obviously superior to the traditional temporary plugging method.

\subsubsection{Dynamic Pollution Experiment}

The experimental cores are from the representative natural cores in Hailaer area, and the experimental process is in accordance with the People's Republic of China Petroleum Industry Standard SY/T6540-2002. Polluting the selected cores by base mud, $1 / 3$ bridging rule, temporary plugging and new temporary plugging method respectively, we got the measured permeability recovery which is showed in Figure 4. It can be seen from Figure 4 that the permeability recovery after pollution by drilling fluid without shielding temporary plugging technology was only $47.20 \%$, and the permeability recovery was greatly improved after temporary plugging. Especially the formulated drilling fluid adopting new temporary plugging method, its permeability recovery was as high as $90.8 \%$. Thus, the new temporary plugging method has a high protection effect for oil layers.

\section{CONCLUSIONS}

New temporary plugging method based on Andreasen equation strictly satisfy mathematical theory, at the same time it considers the fractal characteristics of reservoir pore and grindingbody, and it puts mathematical knowledge into oil industry excellently.

This temporary plugging method is easy to operate, fully taking into consideration of the pore filling of various sizes in reservoirs and the pore filling among temporary plugging particles. It also achieves filling with minimal air voids by means of formulating and optimizing of rigid particles and softening particles

The indoor experiments present that the filtration has

Table 3. Rheological property and filter loss of drilling fluid with Temporary Plugging Agent.

\begin{tabular}{|c|c|c|c|c|c|c|}
\hline $\begin{array}{c}\text { Temporary } \\
\text { Plugging } \\
\text { Agent }\end{array}$ & $\begin{array}{c}\varphi 60 \\
0\end{array}$ & $\begin{array}{c}\varphi 30 \\
0\end{array}$ & $\begin{array}{c}\mathrm{YP} \\
\mathrm{Pa} \\
\mathrm{C}\end{array}$ & $\frac{\mathrm{PV}}{(\mathrm{mPa} \cdot \mathrm{s})}$ & $\frac{\mathrm{FL}}{(\mathrm{mL})}$ & $\mathrm{FL}_{\text {HTHP }}$ \\
\hline Mud & 54 & 32 & 5 & 22 & 4.3 & 10.1 \\
\hline $\mathrm{Mud}+5 \% \mathrm{~A}$ & 59 & 37 & 7.5 & 22 & 2.1 & 6.3 \\
\hline $\mathrm{Mud}+5 \% \mathrm{~B}$ & 62 & 40 & 9 & 22 & 3.2 & 9.3 \\
\hline
\end{tabular}

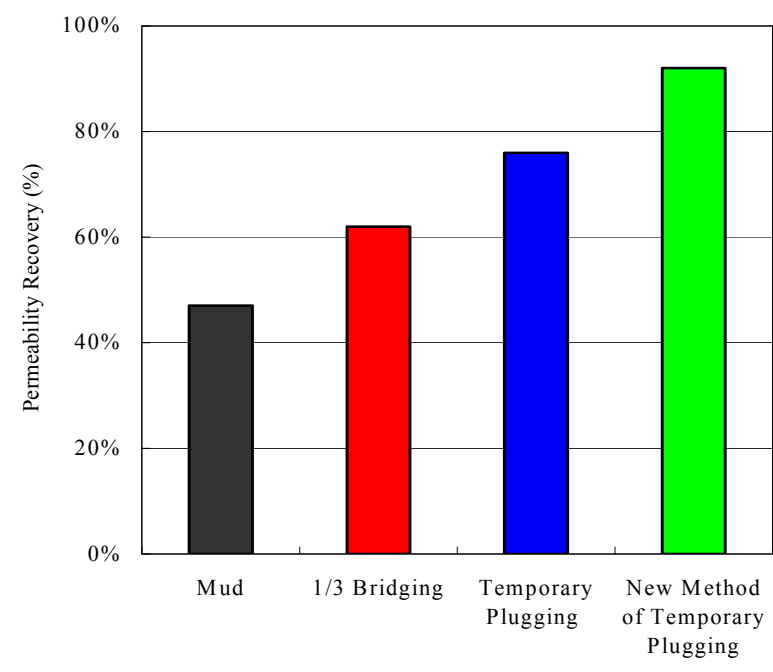

Figure 4. Permeability recovery after pollution by drilling fluid.

significantly reduced after adopting new temporary plugging method based on Andreasen equation and the permeability recovery was greatly improved. This shows that the new temporary plugging method has more ideal effect for protecting reservoirs.

\section{REFERENCES}

[1] Luo, X.D. and Luo, P.Y. (1992) Application and research of temporary plugging technology in reservoir protection. Drilling Fluid \& Completion Fluid, 9(2), 19-27.

[2] Huang, B.Z. (2007) Microscopic mechanisms and model design of close packing theory. Petroleum Drilling Techniques, 35(1), 5-12.

[3] Cai, J.H., Wu, X.M. and Sui, G. (2009) Research on environmentally safe temporarily plugging drilling fluid in water well drilling. Asia Pacific Health, Safety, Security and Environment Conference, Jakarta.

[4] Jiang, G.C., Ji, C.F. and Ma, X.P. (2009) Study and application of reservoir protection fluid system for drilling \& completion using oil-film method. 8th European Formation Damage Conference, Scheveningen, The Netherlands.

[5] Jiang, G.C., Huang L.J. and Zhang G.R. (2006) New technology of unconsolidated sandstone reservoir protection. Publishing House of China University of Petroleum, Dongying.

[6] Zhang, J.B., Yan, J.N. and Zhao, H,Y. (2004) Optimization of bridging particle size distribution of drilling fluid for formation protection. Drilling Fluid \& Completion Fluid, 21, 4-7.

[7] Zeng, F. and Hu, Y. (1995) Particle technology of mineral processing. China University of Mining and Technology Press.

[8] Tang, M., Pan, J. and Ba, H. (2005) The model of the cementing matrix powder group fractal geometry denseness effect. Journal of Shenyang Jianzhu University (Natural Science), 21. 